\title{
Design of a HVCMOS pixel sensor ASIC with on-chip readout electronics for ATLAS ITk upgrade
}

\author{
Mridula Prathapan ${ }^{*} \dagger$ Pierre Barrillon $^{a}$, Mathieu Benoit $^{f}$, Raimon Casanova ${ }^{e}$, Felix \\ Ehrler $^{b}$, Patrick Pangaud ${ }^{a}$, Sourav Pusti ${ }^{d}$, Rudolf Schimassek ${ }^{b}$, Eva Vilella ${ }^{g}$, Alena \\ Weber $^{c, b}$, Winnie Wong ${ }^{f}$, Hui Zhang ${ }^{b}$, Ivan Perić ${ }^{b}$ \\ ${ }^{a}$ Aix Marseille Univ, CNRS/IN2P3, CPPM, Marseille, France \\ ${ }^{b}$ ASIC and Detector Laboratory, Karlsruhe Institute of Technology, Germany \\ ${ }^{c}$ Physikalisches Institut, University Heidelberg, Germany \\ ${ }^{d}$ Department of Physics, IIT Bombay, India \\ ${ }^{e}$ IFAE Institute for High Energy Physics, Barcelona, Spain \\ ${ }^{f}$ Department of nuclear physics, University of Geneva, Switzerland \\ ${ }^{g}$ Department of Physics, University of Liverpool, UK \\ E-mail: mridula.prathapan@kit.edu, ivan.peric@kit.edu
}

\begin{abstract}
ATLASpix is a series of monolithic High Voltage CMOS (HVCMOS) sensor chips that are engineered to meet the requirements of outer layers of ATLAS ITk pixel tracker for HL-LHC upgrade. They are large collection electrode designs on high resistive wafers to ensure high detection efficiency and radiation tolerance. The readout electronics are placed on the chip periphery. ATLASpix1_M2 prototype is fabricated in a commercial $180 \mathrm{~nm}$ CMOS technology and has an active area of $1.6 \mathrm{~cm} \times 0.33 \mathrm{~cm}$. No clock signals are propagated inside the pixel matrix reducing the crosstalk and helping to achieve an estimated power consumption of $300 \mathrm{~mW} / \mathrm{cm}^{2}$. This work presents the design of ATLASpix_M2 with emphasis on its readout electronics, together with some experimental results.
\end{abstract}

Topical Workshop on Electronics for Particle Physics (TWEPP2018)

17-21 September 2018

Antwerp, Belgium

\footnotetext{
${ }^{*}$ Speaker.

$\dagger$ The author would like to thank the DFG-funded Doctoral School "Karlsruhe School of Elementary and Astroparticle Physics: Science and Technology" for their support on the research that led to this work
} 


\section{Introduction}

High Voltage CMOS (HVCMOS) sensor chips could be the building blocks of the proposed CMOS quad module which is a low cost alternative to hybrid modules for ATLAS Inner Tracker (ITk) upgrade. The HVCMOS designs are targeted to meet the specifications [1] of ATLAS ITk pixel barrel layer 4 . The sensors are tested to be radiation tolerant up to at least $100 \mathrm{Mrad}$ and $1 \times 10^{15} \mathrm{n}_{\mathrm{eq}} / \mathrm{cm}^{2}$ [2]. The principle of operation of HVCMOS sensors is explained in [3]. The pixel electronics [4] include a charge sensitive amplifier and a discriminator that are placed inside the n-well which acts as the charge collection electrode. A high voltage is used to deplete the p-substrate underneath. The major blocks of ATLASpix chips are HVCMOS sensor array, hit buffers to store the hit information, Readout Control Unit (RCU) for scheduling the readout operation and several full custom blocks such as PLL, bias block, serializer etc. The signals caused by particle hits are digitized using discriminators and transferred to the digital periphery of the ASIC where the hit buffers are located. The hit buffer acts as a temporary storage for hit information until it is sent to the End of Column (EoC) buffer. There are two design variants based on the readout architecture, namely ATLASpix1_Simple and ATLASpix1_M2. ATLASpix1_Simple employs a traditional column drain readout without trigger whereas ATLASpix1_M2 adopts a novel triggered readout scheme [5]. The architecture of ATLASpix1_Simple and its experimental studies are described in [4]. Both ATLASpix1_Simple and ATLASpix1_M2 share the same pixel electronics and RCU.

\section{Architecture and readout electronics of ATLASpix1_M2}

ATLASpix_M2 employs a parallel hit transfer mechanism from the pixels to hit buffer, which is called "Parallel Pixel to Buffer (PPtB)"[5]. Out of 17920 pixels, each group of 16 pixels forms a super pixel which can be addressed using 8 address lines by projection addressing figure 1 . This helps to save routing space by reducing the number of interconnect lines required to transfer the hit pattern from 16 to 8 . The effect of RC delay of these routing lines that transmit the hit signals from the pixel driver to the hit buffer is investigated. Simulations done on a simplified RC-extracted netlist show a difference of $36 \mathrm{~ns}$ between the RC delays of interconnect lines corresponding to the top most pixel and the bottom most pixel in a column. In order to minimize this effect, the next generation ATLASpix chips are planned to be layouted in a way that the length of routing lines from pixel to hit buffer remain uniform across the matrix. This can be achieved with the help of an additional routing layer. The address encoding scheme shown in figure 1 poses a risk of having ghost hits sharing the same address pattern as real hits. Due to the small area of a super pixel (800 $\times 60 \mu \mathrm{m}^{2}$ ), the rate of multiple clusters is reduced by several orders of magnitude. Assuming a total hit rate of $108 \mathrm{MHz} / \mathrm{cm}^{2}$, the probability of having a hit in the super pixel per bunch crossing is calculated as $1.3 \times 10^{-3}$. Ghost hits are caused either when a single particle produces a cluster on the group edges (figure 1) or when two particles hit the pixels of neighbouring groups in a super pixel. The former scenario leads to a real hit pattern which can be identified as a cluster and two ghost hits that appear as two separate particle hits. Since the probabilty of occurrence of two separate particle hits is much less than than the probability of a single clustered hit, we can neglect the ghost hits during reconstruction. 

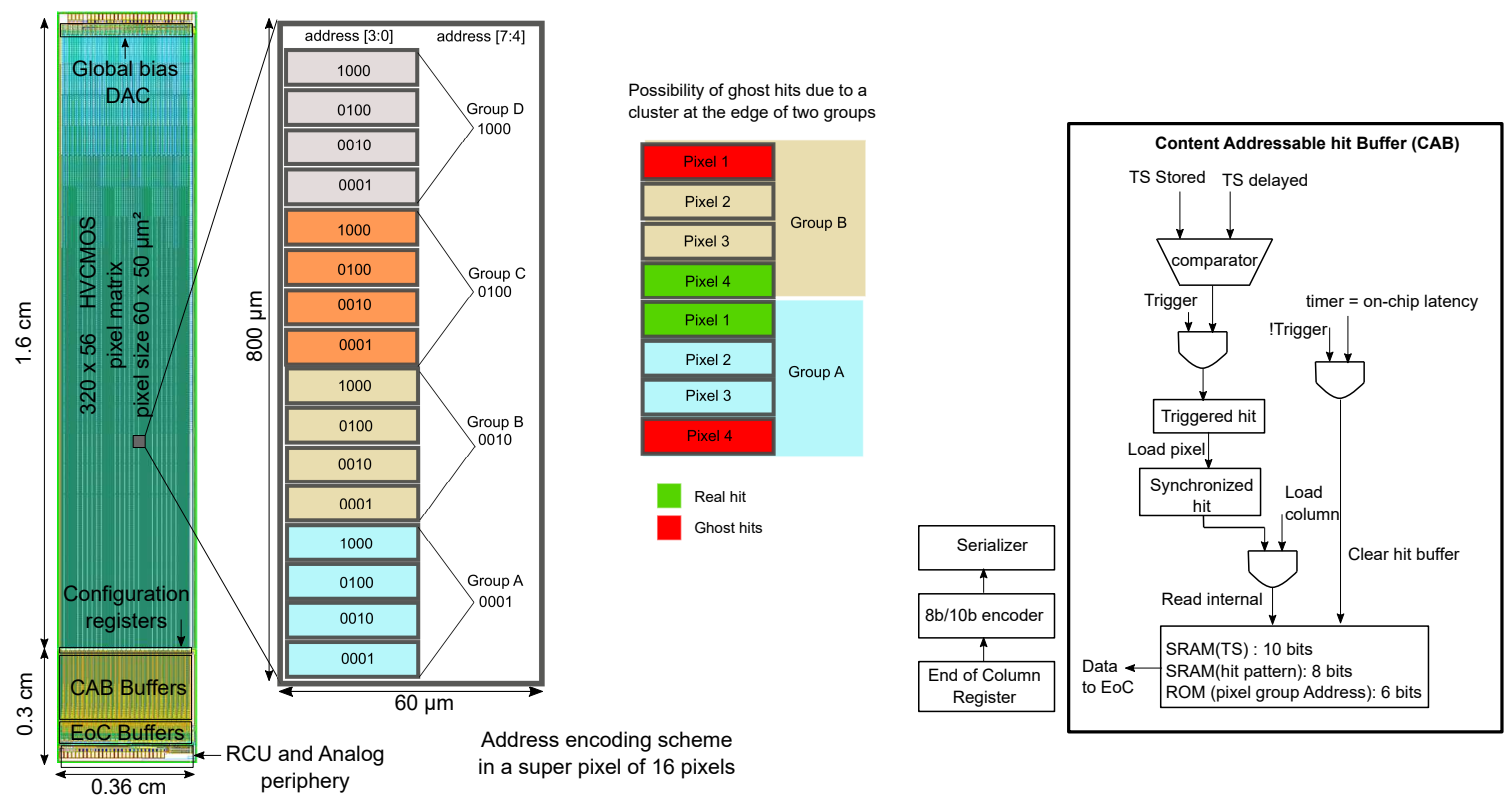

Figure 1: ATLASpix1_M2 Top layout showing address encoding and readout logic

A super pixel is mapped to a Content Addressable hit Buffer (CAB) buffer block which can store four hits. The hit buffer compares the stored Time Stamp (TS) (10-bit) with a delayed TS propagated within the chip, hence it is content addressable. The group address (5-bit) of the super pixel is programmed in address ROM. When there is a particle hit in one or more of the 16 pixels, two or more address lines will be set to high. Therefore, a hit can be detected by calculating logical OR function of the address lines (HitOR). When the HitOR signal goes high, the hit pattern (8-bit) is recorded and transfered to CAB buffer. The time stamp of the corresponding HitOR signal is recorded in the RAM. The time stamps are 10-bit gray coded signals with a period of 25 ns that corresponds to the bunch crossing (BC) period of LHC. The hit information is held in the CAB buffer until its retention time elapses. The retention time is programmable and is called on-chip latency. The on-chip latency is determined by comparing the stored time stamp with an additional time stamp signal that has the same period but different phase as the original time stamp signal. Both time stamps are generated on chip. If level-1 trigger signal is received within the time period before the on-chip latency expires, the stored hits are marked for readout. The hits that are not marked for readout are deleted from the buffer.

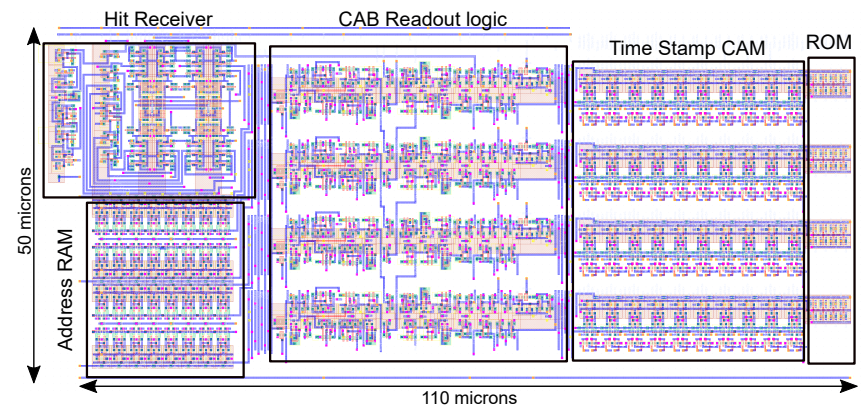

Figure 2: Content Addressable Buffer (CAB) block full custom layout 


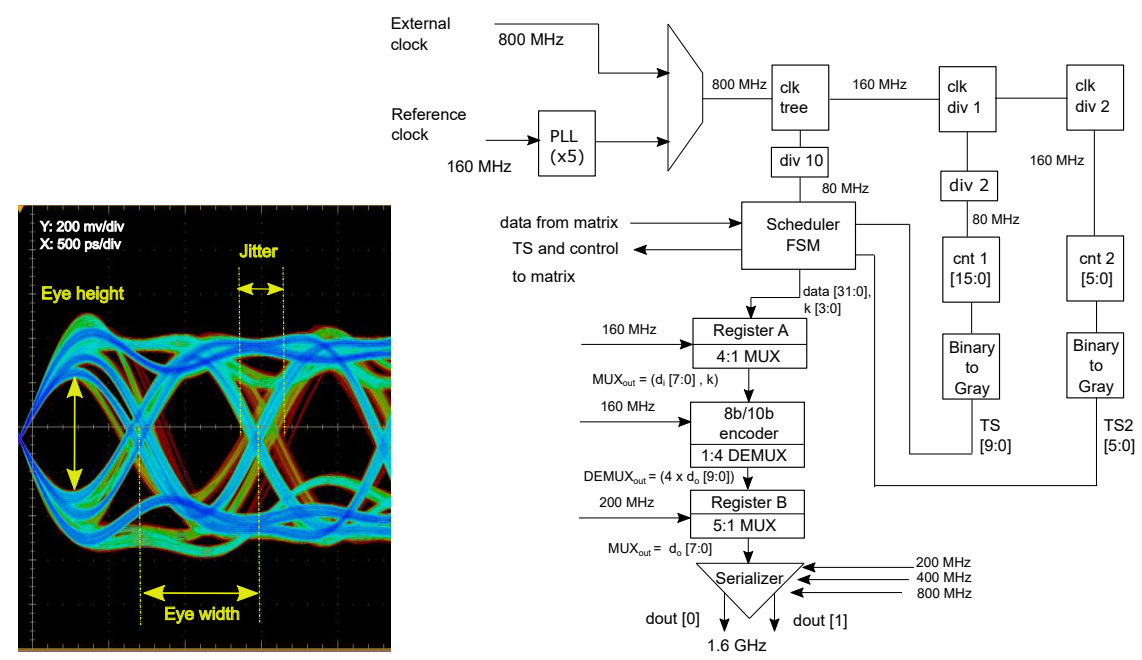

Figure 3: ATLASpix1_M2 Readout Control Unit block and eye diagram at 1.28 Gbps

The readout control unit (figure 3) generates control signals to schedule the entire readout operation. It includes loading of the hit information from the hit buffer to the EoC buffer (load column) and reading the hit data from the $\mathrm{EoC}$ (read column). The hit data is then encoded and serialized. The RCU generates gray-coded time stamps and delayed time stamps (delay value equals on-chip latency) of 10-bits length. The 6-bit TS2 time stamp in figure 3 is unused in ATLASpix1_M2 chip and is used to store time-over-threshold in ATLASpix_Simple chip since both the chips have the same RCU design. The RCU works on multiple clock domains. The clocks of frequencies $160 \mathrm{MHz}, 200 \mathrm{MHz}$ and $400 \mathrm{MHz}$ are generated from an input clock of $800 \mathrm{MHz}$ using johnson counters and combinational logic. The encoder is a pipelined custom $8 \mathrm{~b} / 10 \mathrm{~b}$ encoder with running disparity based on [6]. The serializer is based on a 3-stage MUX tree with input synchronization. The serializer in RCU outputs two bits. The final stage of serialization is achieved using a full custom serializer based on current mode logic.

\section{Measurement results of ATLASpix1_M2}

The data transfer characteristics has been studied with the help of an eye diagram. The oscilloscope probe was connected to the data line on the PCB which is about $10 \mathrm{~cm}$ long. On-chip PLL was used to generate the clock at $800 \mathrm{MHz}$. The serial data output works with Double Data Rate (DDR) of $1.28 \mathrm{Gbps}$ with an eye height of $504 \pm 1 \mathrm{mV}$, an eye width of $580 \pm 1$ ps and a jitter of $100 \pm 0.2 \mathrm{ps}$ as shown in figure 3 . A threshold scan was done over the entire pixel matrix of size $320 \times 56$ at a readout speed of $800 \mathrm{Mbps}$. The trigger is generated a with a fixed delay after the injection, and it has a width of $400 \mathrm{~ns}$ (equivalent to $16 \mathrm{BC}$ ). The on-chip latency is adjusted so that all hits generated by injection are triggered. The injection voltage was then varied from $0 \mathrm{~V}$ to $0.6 \mathrm{~V}$ in steps of $0.025 \mathrm{~V}$ keeping the injection delay, the number of injections (10) and the on-chip latency (43 time stamps, where each time stamp corresponds to $1 \mathrm{BC}$ ) fixed. It is possible to adjust the threshold of every pixel using a 3-bit D/A converter (tune DAC). These tune bits are stored in the pixel memory. The threshold dispersion was reduced by a factor of four after tuning as shown 
in figure 4. The mean threshold is $1055 \mathrm{e}^{-}$with a standard deviation of $35 \mathrm{e}^{-}$. The mean value of noise distribution over the entire pixel matrix after tuning is $78 \mathrm{e}^{-}$.
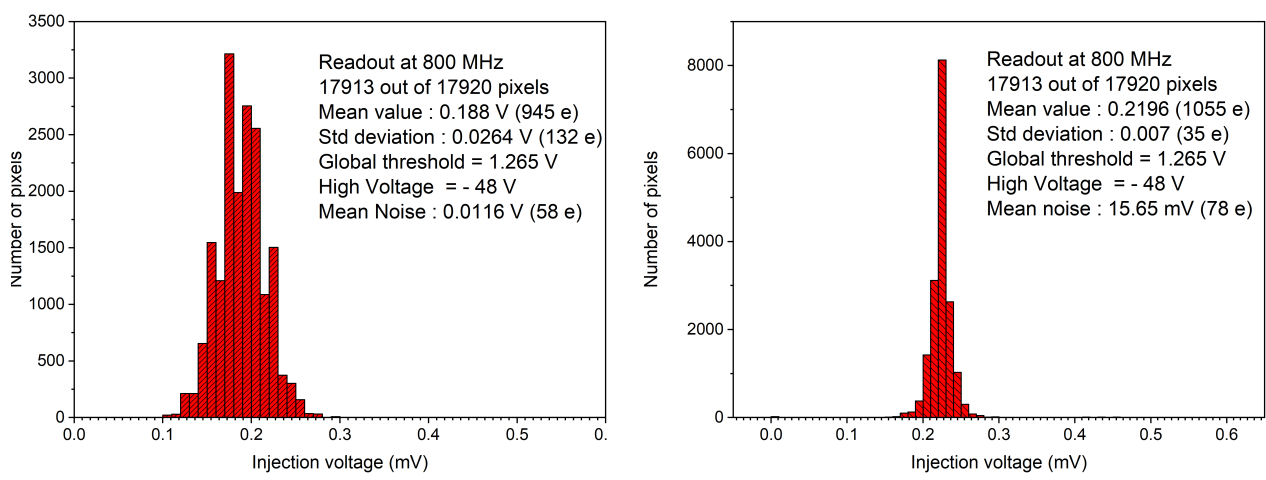

Figure 4: Threshold dispersion before tuning (left) and after tuning (right)

\section{Conclusions and future work}

ATLASpix1_M2 is the first large area and full height HVCMOS sensor prototype with triggered readout. The chip architecture includes several novel design concepts such as the Parallel Pixel to Buffer (PPtB) transfer, address compression of a super pixel, Content Addressable Buffer (CAB) readout, pipelined $8 \mathrm{~b} / 10 \mathrm{~b}$ data encoding and serializer tree. The sensor is fully functional. The thresholds have been measured using the full readout chain. The threshold after tunning had a mean value of $1055 \mathrm{e}^{-}$with a standard deviation of $35 \mathrm{e}^{-}$. The mean value of noise was about $78 \mathrm{e}^{-}$. These values are small compared to the average signal of about $5000 \mathrm{e}^{-}$. The serial data link works at the required data rate of $1.28 \mathrm{Gbps}$. Based on ATLASpix 1 designs, a full size $2 \mathrm{~cm} \times 2 \mathrm{~cm}$ HVCMOS prototype will be developed. Test beam and irradiation studies are planned within the collaboration of six different institutions.

\section{References}

[1] ATLAS Collaboration, "CERN Letter of Intent for the Phase-II Upgrade of the ATLAS Experiment, Technical Report", CERN-LHCC-2012-022, LHCC-I-023, Dec. 2012.

[2] M. Benoit et al. Testbeam results of irradiated ams H18 HV-CMOS pixel sensor prototypes, 2018 JINST 13 P02011

[3] I. Perić, A novel monolithic pixelated particle detector implemented in high-voltage CMOS technology, Nucl. Instrum. Meth. A582 (2007) 876-885.

[4] I. Perić et al. A high-voltage pixel sensor for the ATLAS upgrade, Nuclear Inst. and Methods in Physics Research, A (2018), https://doi.org/10.1016/j.nima.2018.06.060

[5] M.Prathapan et al. Towards the large area HVCMOS demonstrator for ATLAS ITk, Nuclear Inst. and Methods in Physics Research, A (2018), https://doi.org/10.1016/j.nima.2018.11.022

[6] A. X. Widmer, P. A. Franaszek A DC-balanced, partitioned-block, 8B/10B transmission code, IBM Journal of Research and Development, v.27 n.5, p.440-451, September 1983 [doi $>10.1147 / \mathrm{rd} .275 .0440]$ 